\title{
Análise da força e autonomia de idosas: relação entre idade e performance musculoesquelética
}

\author{
Anita Graziela Souza Vieira*; Ludmila Schettino"; Marco Machado"*; Rafael Pereira ${ }^{\text {"**** }}$
}

\section{Resumo}

O envelhecimento acarreta alterações na autonomia, na força e na composição corporal dos indivíduos, com destaque para a sarcopenia. O presente estudo teve como objetivo analisar a relação entre a idade e a performance musculoesquelética de idosas. Sessenta e três idosas foram submetidas a uma avaliação antropométrica, do pico de força de preensão manual e do desempenho em testes de autonomia funcional (levantar-se e sentar-se em 30s e levantar-se e andar $3 \mathrm{~m}$ ). Foi verificada a correlação entre a idade, desempenho na força e nos testes de autonomia e a comparação da força e do desempenho nos testes aplicados entre três grupos, divididos por faixa etária (50-64, 65-74 e 75-86 anos). Observou-se correlação positiva entre a idade e tempo demandado para realizar o teste de levantar-se e andar $3 \mathrm{~m}$, correlação negativa entre a variável idade e o pico de força de preensão manual e entre a idade e o número de repetições realizadas durante o teste de levantar-se e sentar-se em 30s. Houve diferença significativa $(p<0,05)$ no pico de força de preensão manual entre as faixas etárias de 50-64 anos e 75-86 anos, sendo o maior no primeiro grupo, e diferença significativa no desempenho do tes- te de levantar-se e andar, com o desempenho pior no grupo 75-86 anos. De acordo com os resultados, o envelhecimento leva à diminuição da performance musculoesquelética. Por isso, é recomendada a aplicação de testes funcionais rotineiramente, com o intuito de identificar os diferentes graus de comprometimento funcional em indivíduos idosos, visando nortear ações preventivas e terapêuticas.

Palavras-chave: Envelhecimento. Força Muscular. Saúde do Idoso.

\section{Introdução}

O declínio das funções orgânicas é uma característica do envelhecimento, e o comprometimento da função musculoesquelética é a principal causa do aumento do risco de quedas e, por consequência, das morbidades advindas da imobilização no leito. Essa condição interfere negativamente na capacidade ou autonomia funcional do idoso, resultando em declínio da qualidade de vida, uma vez que tende a se tornar mais dependente para a realização de suas

* Discente do curso de Fisioterapia da Universidade Iguaçu (Campus V).

** Especialista em Fisiologia do Exercício.

**** Docente da Universidade Iguaçu (Campus V); mestre em Ciência da Motricidade Humana.

**** Universidade Estadual do Sudoeste da Bahia, Departamento de Ciências Biológicas, Rua José Moreira Sobrinho, s/n, Bairro Jequiezinho, CEP 45210-506, Jequié - BA. E-mail: rafaelpereira@brjb.com.br

$\longrightarrow$ Recebido em março de 2009 - Avaliado em março de 2009.

$\rightarrow$ doi:10.5335/rbceh.2009.021 
atividades básicas do cotidiano. (KAUFFMAN, 2001; DAVINI; NUNES, 2003; DESCHENES, 2004).

Durante o processo de envelhecimento, podem-se observar modificações desfavoráveis no que diz respeito aos componentes da aptidão muscular, resultando em alterações na forma de andar, com aumento do tempo necessário para se percorrer uma distância determinada, gerando uma maior necessidade de apoio para o deslocamento. (FARINATTI; LOPES, 2004).

De acordo com Monteiro et al. (2004), a atividade física e laborativa funciona como um forte estímulo para o metabolismo hormonal e, consequentemente, para uma melhora da saúde funcional, das atividades de vida diária (AVD) e da qualidade de vida dos gerontes, tornando o envelhecimento bem-sucedido. Outros autores (HUGHES et al., 2002; ROUBENOFF, 2001; MATSUDO et al., 2002; ACMS, 2003) também apontam que o exercício físico é uma forma eficaz de atenuar a perda muscular e de melhorar o equilíbrio e a mobilidade que acompanham o envelhecimento, reduzindo o risco de quedas e lesões e promovendo uma melhor autonomia funcional.

A prática regular de atividade física também contribui para a melhora ou manutenção da capacidade cardiorrespiratória, da flexibilidade, coordenação, agilidade e força muscular, sendo a manutenção desta última fundamental para a capacidade de locomoção e realização de AVDs. (FARINATTI; LOPES, 2004). Dessa forma, o presente estudo objetivou analisar a relação entre a idade, a performance musculoesquelética e a autonomia de idosas residentes na comunidade.

\section{Materiais e métodos}

\section{Amostra}

Participaram do estudo 63 indivíduos do gênero feminino, com idade entre $50 \mathrm{e}$ 86 anos (70 \pm 7 anos), aptas fisicamente a realizar a bateria de testes selecionada para a avaliação da autonomia funcional e independentes no desempenho das atividades diárias. As participantes, residentes na cidade de Carangola/MG, preencheram um termo de participação consentida de acordo com a resolução $\mathrm{n}^{0}$ 196/1996, de 10 de outubro de 1996, do Conselho Nacional de Saúde, sobre as recomendações éticas referentes à realização de pesquisas envolvendo seres humanos. Foi considerada como critério de exclusão qualquer tipo de condição aguda ou crônica que pudesse comprometer ou fosse um fator de impedimento para a realização dos testes.

\section{Procedimentos e instrumentos}

Primeiramente, foi aferida a pressão arterial de cada participante. Em seguida, foram colhidos os dados antropométricos, como massa corpotal total $(67 \pm$ $15 \mathrm{~kg})$, estatura $(151 \pm 6 \mathrm{~cm})$, índice de massa corporal $\left(30 \pm 7 \mathrm{~kg} / \mathrm{cm}^{2}\right)$, perimetria de braço (direito: $30 \pm 5$; esquerdo: $30 \pm 5 \mathrm{~cm}$ ), antebraço (direito: $23 \pm 2$; esquerdo: $23 \pm 2 \mathrm{~cm}$ ), coxa (direita: $56 \pm$ 9; esquerda: $56 \pm 9 \mathrm{~cm}$ ) e perna (direita: $35 \pm 3$; esquerda: $35 \pm 3 \mathrm{~cm}$ ). As médias de perímetro foram realizadas na porção medial de cada segmento.

Para análise da força e da autonomia foram coletados dados referentes, respectivamente, à força de preensão manual e 
ao desempenho nos testes de levantar-se e sentar-se em 30s (MORROW et al., 2003) e levantar-se e andar $3 \mathrm{~m}$ (TUG). (MORROW et al., 2003; SHUMWAYCOOK; WOOLLACOTT, 2003; SOARES et al., 2002). Os testes foram realizados duas vezes cada, com intervalo de um minuto entre cada tentativa e dois minutos entre os testes, sendo considerado o melhor desempenho nas duas tentativas para posterior análise.

Para coleta da força de preensão manual foi utilizado um transdutor de força tração-compressão (EMG System, Brasil) com range de 0-200 kgf, fixado a um aparato construído especialmente para quantificar da força de preensão. A força de preensão foi coletada com o indivíduo sentado e tendo o braço ao longo do corpo e o cotovelo fletido a $90^{\circ}$; a duração da contração foi de $5 \mathrm{~s}$. $O$ teste foi realizado duas vezes, com intervalo de um minuto entre as tentativas, sendo considerado para análise o maior pico de força de preensão obtido entre as duas tentativas.

\section{Análise estatística}

Foi realizado o teste de normalidade de Shapiro-Wilk com nível de significância de $\mathrm{p}<0,05$, sendo verificado que os dados de idade, pico de força de preensão e desempenho dos testes apresentaram distribuição normal. Os dados de idade foram correlacionados com as variáveis pico de força de preensão manual e desempenho nos testes de levantar-se e sentar-se em 30s e levantar-se e andar 3 $\mathrm{m}$ por meio da correlação de Pearson com nível de significância de $\mathrm{p}<0,05$.
Para verificar o efeito do envelhecimento sobre a funcionalidade, a amostra foi dividida em Quartis, o que gerou uma divisão por faixa etária em aproximadamente três décadas (50-64, 65-74, 75-86 anos). Foi aplicado o teste de normalidade de Shapiro-Wilk para avaliação da normalidade dos dados para cada grupo, com nível de significância de $\mathrm{p}<0,05$. Como as variáveis pico de força de preensão e desempenho no teste de levantar-se e andar $3 \mathrm{~m}$ não apresentaram distribuição normal no grupo 65-74 anos, foi aplicado o teste de Kruskal-Wallis com nível de significância de $\mathrm{p}<0,05$, para avaliar a igualdade ou não do desempenho físico dos idosos em diferentes faixas etárias.

\section{Resultados}

A análise da correlação de Pearson demonstrou que a variável idade correlaciona-se positivamente com o tempo demandado no teste de levantarse e andar $3 \mathrm{~m}\left(\mathrm{r}^{2}=0,45\right.$ sendo $\left.\mathrm{p}<0,05\right)$ e negativamente com o pico de força de preensão manual $\left(r^{2}=-0,38\right.$ sendo $\mathrm{p}<0,05)$ e com o desempenho no teste de levantar-se e sentar-se em 30 s $\left(r^{2}=-0,36\right.$ sendo $\left.\mathrm{p}<0,05\right)$.

Observou-se, pela comparação entre as faixas etárias, diferença significativa para o pico de força de preensão quando comparadas as faixas etárias de 50-64 $(24 \pm 1 \mathrm{~kg})$ e $75-86$ anos $(19 \pm 1 \mathrm{~kg})$ ( $\mathrm{p}<0$ 05), tendo o grupo 50-64 maior pico de força; não houve diferença da força do grupo 65-74 anos (22 $\pm 1 \mathrm{~kg})$ na comparação com os demais grupos ( $p>0,05)$ (Fig. 1). 


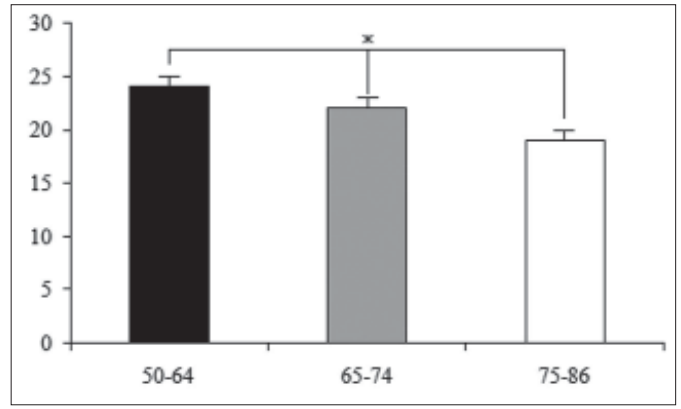

Figura 1 -Média \pm desvio-padrão do pico de força de preensão (kgf) entre as faixas etárias estudadas. (*) Diferença significativa entre as faixas etárias 50-64 e 75-86 ( $<<0,05)$.

O desempenho no teste de levantarse e andar demonstrou diferença significativa entre os grupos 50-64 (8,6 5 0,4s) e 75-86 anos $(10,7 \pm 0,3 \mathrm{~s})(\mathrm{p}<0,05)$ e entre os grupos 65-74 (9,7 $\pm 0,4 \mathrm{~s})$ e $75-86$ anos ( $\mathrm{p}<0,05)$, não havendo diferença significativa entre os grupos 50-64 e 65$74 \operatorname{anos}(p>0,05)$ (Fig. 2).

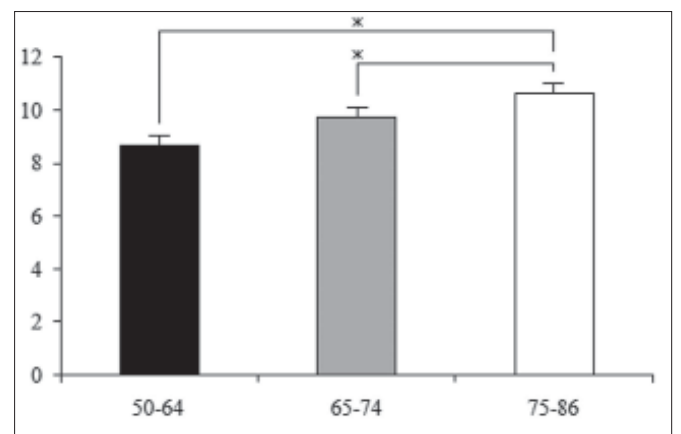

Figura 2 -Média \pm desvio-padrão do desempenho no teste de tempo de levantar e andar (TUG) entre as faixas etárias estudadas. (*) Diferença significativa entre as faixas etárias 50-64 e 75-86 e entre as faixas etárias 65-74 e 75$86(\mathrm{p}<0.05)$.
Não foi observada diferença significativa entre os grupos para o desempenho no teste de levantar-se e sentar-se em 30s (50-64: $15 \pm 1$ repetições / 65-74: $14 \pm 1$ repetições / 75-86 anos: $13 \pm 1$ repetições) ( $\mathrm{p}>0,05)$ (Fig. 3).

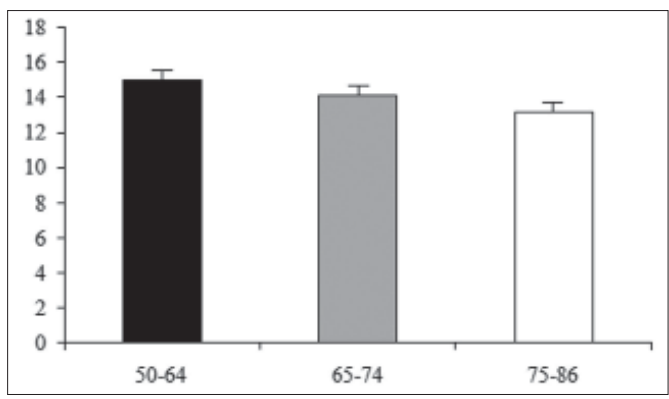

Figura 3 -Média \pm desvio-padrão do desempenho do número de execuções no teste de levantar-se e sentar-se em 30s entre as faixas etárias estudadas. Não houve diferença significativa entre as faixas etárias estudadas $(p>0,05)$.

\section{Discussão}

O presente estudo teve o objetivo de avaliar a autonomia funcional de idosas residentes na comunidade. Para a avaliação foram utilizados testes já descritos na literatura, como teste de preensão manual, teste de levantar-se e sentar-se em 30s e teste de levantar-se e andar $3 \mathrm{~m}$.

Nossos resultados apontam o declínio da autonomia funcional como consequência do envelhecimento, o que pode ser inferido pelo declínio no pico de força de preensão manual associado ao envelhecimento. Lauretani et al. (2003) e Geraldes et al. (2008) afirmam que a medida da força de preensão é o melhor indicador de declínio do sistema mus- 
culoesquelético em idosos - sarcopenia - e do desempenho funcional de idosos.

A redução da força associada ao envelhecimento é bem descrita na literatura (CARVALHO; SOARES, 2004; LACOURT; MARINI, 2006), sendo justificada pela redução do número e do tamanho das fibras musculares, principalmente das fibras do tipo II, também chamadas de "fibras de contração rápida" e que produzem grande quantidade de força. (DESCHENES, 2004). No presente estudo observou-se uma diferença de $21 \%$ no pico de força entre os grupos de faixa etária de 50-64 anos e o grupo 75-86 anos, a qual pode ser justificada pela redução das fibras musculares do tipo II associada ao envelhecimento, uma vez que o pico de força representa a força máxima gerada num determinado período de tempo, corroborando, assim, com os dados existentes na literatura. (HAËKKINEN et al., 2001; DESCHENES, 2004).

A amostra aqui estudada foi constituída apenas por mulheres, no entanto estudos anteriores apontam para uma redução na força de preensão associada ao envelhecimento tanto para homens quanto para mulheres. (MACLENNAN et al., 1980; GIAMPAOLI et al., 1999).

$\mathrm{O}$ teste de levantar-se e sentar-se em 30 s é descrito na literatura como um instrumento que avalia a força dos membros inferiores (RIKILI; JONES, 1999). Foi observada neste estudo uma correlação negativa entre a idade e 0 desempenho neste teste, o que indica uma diminuição desta força de membros inferiores associada ao envelhecimento. $\mathrm{Na}$ comparação entre as faixas etárias analisadas não houve diferença significativa, o que pode ser justificado pela falta de precisão do método, que mensura a força de modo indireto e depende de outras variáveis, como flexibilidade de várias articulações e da compreensão do avaliado, diferentemente das medidas de força realizadas por meio de sistemas de dinamometria, como foi medida a força de preensão. Além disso, a medida da força de membros inferiores pode não ser o melhor indicador de declínio muscular e funcional associado ao envelhecimento, o que é atribuído à medida da força de preensão, como citado anteriormente. (LAURETANI et al., 2003); GERALDES et al., 2008).

Estudos evidenciam que a força muscular atinge seu pico por volta dos trinta anos de idade, com um grau bem mais rápido de diminuição após os sessenta anos (KAUFFMAN, 2001). O prejuízo da função pode ser considerado um dos principais fatores que interferem na qualidade de vida do idoso. (LACOURT; MARINI, 2006).

$\mathrm{O}$ teste de levantar-se e andar $3 \mathrm{~m}$ é utilizado para mensurar a mobilidade em idosos (PODSIADLO et al., 1999; SHUMWAY-COOK et al., 2000; MACIEL; GUERRA, 2005), sendo descrito como um preditor de risco de quedas para esses. (SHUMWAY-COOK et al., 2000; BISCHOFF et al., 2003). Os resultados do presente estudo demonstram correlação positiva entre a idade e o tempo demandado no teste de levantarse e andar, indicando um declínio na potência muscular, velocidade, agilidade e equilíbrio dinâmico, variáveis avaliadas neste teste. (PODSIADLO et al., 1999; BISCHOFF et al., 2003). 
A comparação do desempenho do teste de levantar-se e andar entre as faixas etárias estudadas indica um declínio no desempenho com o passar das décadas. Diferentemente dos demais testes utilizados neste estudo, o teste de levantar-se e andar demonstrou um aumento significativo no tempo gasto para executar o teste, sendo $13 \%$ maior no grupo 65-74 e de 24\% no grupo 75-86 anos, quando comparado ao grupo 50-64 anos, e $10 \%$ maior, quando comparados os grupos de faixa etária de 65-74 e 7586 anos. Este resultado pode indicar uma maior sensibilidade deste teste em detectar o declínio funcional em idosos, já que avalia múltiplas qualidades físicas, principalmente o equilíbrio dinâmico, e é relacionado ao risco de quedas e avaliador da autonomia em idosos. (PODSIADLO; RICHARDSON, 1991; SHUMWAY-COOK et al., 2000; BISCHOFF et al., 2003).

A atividade física pode influenciar de modo positivo no decréscimo de força dos membros inferiores associado ao envelhecimento, diminuindo o risco de quedas na população idosa, de acordo com a relação que existe entre as variáveis de força dos membros inferiores, desabilidades e risco de quedas referenciadas na literatura. (RIKLI; JONES, 1999).

\section{Conclusão}

Os resultados deste estudo permitem concluir que o envelhecimento acarreta um declínio progressivo da performance musculoesquelética, o que pode comprometer a autonomia de indivíduos idosos. Conclui-se ainda que a aplicação de testes funcionais rotineiros pode ajudar na estratificação de idosos com menor autonomia e, portanto, com maior risco de quedas e dependência nas tarefas, 0 que pode nortear ações preventivas e terapêuticas em idosos.

\section{Agradecimentos}

Agradecemos a Cátia Roriz Bocard Fialho, Silvya de Lima Filó e Rodolfo Corrêa Novaes de Souza por viabilizarem e colaborarem na coleta dos dados.

Analysis of the strength and autonomy of old women: relationship between age and muscle performance

\section{Abstract}

The aging carts to alterations in the autonomy, strength and body composition of the elderlies, with focus in the sarcopenia. The objective of present study was to analyze the relationship between the age and muscular performance in elderly women. Sixty-three elderly women were submitted to an anthropometrical evaluation, measure of handgrip strength and evaluation of the performance in functional tests ("30-Second chair stand" and "Timed get up and go"). It were verified the correlation among age, handgrip strength and performance in functional tests, besides this, it were done the comparison of the handgrip strength and performance among three groups of elderly grouped by age (50-64, 65-74 e 75-86 years). Positive correlation was observed between age and demanded time to execute the "Timed get up and go" test, and negative correlation were observed between age and handgrip strength and between age and performance in "30-Second Chair Stand" test. There were significant difference in the handgrip 
strength and performance in the "Timed get up and go" test among groups of elderly of 50-64 years and 75-86 years, being observed greater results in the first group. Our results indicate a decrease in muscular performance associated to the aging, being recommended the use of functional test routinely to identify different degrees of functional deficiency of elderly and guide preventive and therapeutic actions.

Key words: Aging. Muscle Strength. Health of the Elderly.

\section{Referências}

AMERICAN COLLEGE OF SPORTS MEDICINE. Diretrizes do ACSM para os testes de esforço e sua prescrição. 6. ed. Rio de Janeiro: Guanabara Koogan, 2003.

BISCHOFF, H. A. et al. Identifying a cut-off point for normal mobility: a comparison of the timed 'up and go' test in community-dwelling and institutionalised elderly women. Age and Ageing, v. 32, p. 315-320, 2003.

CARVALHO, J.; SOARES, J. M. C. Envelhecimento e força muscular: breve revisão. Revista Portuguesa de Ciências do Desporto, v. 4, n. 3, p. 79-93, 2004.

DAVINI, R.; NUNES, C. V. Alterações no sistema neuromuscular decorrentes do envelhecimento e o papel do exercício físico na manutenção da força muscular em indivíduos idosos. Revista Brasileira de Fisioterapia, São Carlos, v. 7, n. 3, p. 201-207, 2003.

DESCHENES, M. R. Effects of aging on muscle fibre type and size. Sports Medicine, v. 34, n. 12, p. 809-824, 2004.

FARINATTI, P. T. V.; LOPES, L. N. C. Amplitude e cadência do passo e componentes de aptidão muscular em idosos: um estudo correlacionado multivariado. Revista Brasileira de Medicina do Esporte, São Paulo, v. 10, n. 5, p. 389-394, 2004.
GERALDES, A. A. R. et al. A força de preensão manual é boa preditora do desempenho funcional de idosos frágeis: um estudo correlacional múltiplo. Revista Brasileira de Medicina do Esporte, São Paulo, v. 14, n. 1, p. 12-16, 2008.

GIAMPAOLI, S. et al. Hand-grip strength predicts incident disability in non-disabled older men. Age and Ageing, v. 28, p. 283-288, 1999.

HAËKKINEN, K. et al. Changes in electromyographic activity, muscle fibre and force production characteristics during heavy resistance/power strength training in middleaged and older men and women. Acta Physiol Scand, v. 171, p. 51-62, 2001.

HUGHES, V. A. et al. Longitudinal changes in body composition in older men and women: role of body weight change and physical activity. Am. J. Clin. Nutr., v. 76, p. 473-481, 2002.

KAUFFMAN, T. L. Manual de reabilitação geriátrica. Rio de Janeiro: Guanabara Koogan, 2001.

LACOURT, M. X.; MARINI, L. L. Decréscimo da função muscular decorrente do envelhecimento e a influência na qualidade de vida do idoso: uma revisão de literatura. Revista Brasileira de Ciências do Envelhecimento Humano, Passo Fundo, v. 1, n. 2, p. 114-121, jul./dez. 2006.

LAURETANI, F. et al. Age-associated changes in skeletal muscles and their effect on mobility: an operational diagnosis of sarcopenia. J. Appl. Physiol., v. 95, p. 1851-1860, 2003.

MACIEL, A. C. C.; GUERRA, R. O. Fatores associados à alteração da mobilidade em idosos residentes na comunidade. Revista Brasileira de Fisioterapia, São Carlos, v. 9, n. 1, p. 17-23, 2005.

MACLENNAN, W. J. et al. Is weakness in old age due to muscle wasting? Age and Ageing, v. 9, p. 188-192, 1980. 
MATSUDO, V. K. et al. Promotion of physical activity in a developing country: the agita São Paulo experience. Public Health Nutrition, v. 5, n. 1A, p. 1-10, 2002.

MONTEIRO, N. et al. Efeitos de um programa de atividade física regular sobre os níveis séricos basais de IGF em idosas. Revista Fitness \& Performance Journal, v. 3, n. 3, p. 130-135, 2004.

MORROW, J. R. et al. Medida e avaliação do desempenho humano. 2. ed. Porto Alegre: Artmed, 2003.

PODSIADLO, D.; RICHARDSON, S. The timed "Up \& Go": a test of basic functional mobility for frail elderly persons. J. Am. Geriatr. Soc,. v. 39, p. 142-148, 1991.

RIKLI, R. E.; JONES, C. J. Development and validation of a functional fitness test for community-residing older adults. Journal of Aging and Physical Activity, v. 7, p. 129-161, 1999.

ROUBENOFF, R. Origins and clinical relevance of sarcopenia. Can. J. Appl. Physiol., v. 26, p. $78-89,2001$.

SHUMWAY-COOK, A.; WOOLLACOTT, M. H. Controle motor: teoria e aplicações práticas. 2. ed. São Paulo: Manole, 2003.

SOARES, A. V. et al. Estudo comparativo sobre a propensão de quedas em idosos institucionalizados e não-institucionalizados através do nível de mobilidade funcional. Fisioterapia Brasil, São Paulo, v. 4, n. 1, p. 13-16, 2002. 